\title{
Localized Surface Plasmon Resonance Sensing of Nanoparticles
}

\author{
R. HORCHANI* \\ Department of Physics, College of Sciences, Sultan Qaboos University, \\ P.O. Box 36, PC 123, Muscat, Sultanate of Oman
}

(Received December 2, 2019; revised version January 23, 2020; in final form February 4, 2020)

\begin{abstract}
Localized surface plasmon resonance system using a glass prism and a multi-layered chip is proposed as a detection of silver nanoparticles. The model is robust and very general. It can be used to alter different parameters of the sensor in a way that enables us to optimize its response for a particular analyte. We show that the use of chromium adhesion layers with a graphene layer at the silver-sensing interface leads to the detection of silver nanoparticles from their plasmonic amplitude (self-sensing).
\end{abstract}

DOI: 10.12693/APhysPolA.137.1121

PACS/topics: $61.46 .-\mathrm{w}$

\section{Introduction}

Since the first observation of surface plasmon resonance (SPR) sensor by Wood [1], a large number of applications have been found in areas of medical sciences, biochemistry, and biology, due to its real-time, label-free and noninvasive nature. Recently, there has been a great interest in the use of magnetic and plasmonic nanoparticles as active materials for biomolecule detection [2]. The optical sensor that is based on SPR [3, 4] has been widely used to detect biomolecules due to their sensitivity, label-free assay, and real-time detection [5]. This detection method is very sensitive to size and shape of nanoparticles, and is relied on the changes of the refractive index of the medium surrounding the metal thin film. A biomolecule coming into contact with the thin metal film is adsorbed onto its surface. This leads to increase of the refractive index at the interface and hence the change of the resonance angle. Through this change it is possible to detect the presence of an analyte and its concentration. Many detection techniques have been used, such as measurement of the resonant angle of incidence of light [6,7], measurement of the intensity of the reflected light $[8,9]$, and measurement of the resonant wavelength of the incident light [10].

In this paper, we propose a sensor that mechanism is based on localized surface plasmon resonance, and use for it a glass prism and a combination of different metal layers as tool elements. To calculate the attenuated total reflection (ATR) of the proposed configuration we apply, the so-called, angular interrogation method. It is worth noting that other methods based on wavelength, phase, and intensity can be used as well [11].

*e-mail: ridha.horchani@yahoo.fr

\section{Model}

The Kretschmann configuration (KC) is one of the first setups, with which surface plasmon polariton (SPP) resonances were observed [12]. This configuration along with the Otto configuration was used for measuring dielectric permittivities. In the KC, shown in Fig. 1, a metal film and the material under investigation (analyte) are deposited on the base of a dielectric prism. A beam of $p$-polarized light is transmitted through the prism. Since the dielectric permittivity of the prism $\epsilon_{p}$, is greater than that of the analyte, there is a critical angle at which total internal reflection occurs. At angle greater than the angle of total internal reflection, a sharp minimum is observed in the reflection coefficient due to losses in the metal. This phenomenon is commonly referred to as attenuated total reflection (ATR) [13]. To calculate the ATR, we use a mathematical model based upon transfer matrix method (TMM) [14]. Since the wave propagation is described by $p$ - and $s$-polarized modes for homogeneous materials, the transfer matrix is of rank 2 in this case.

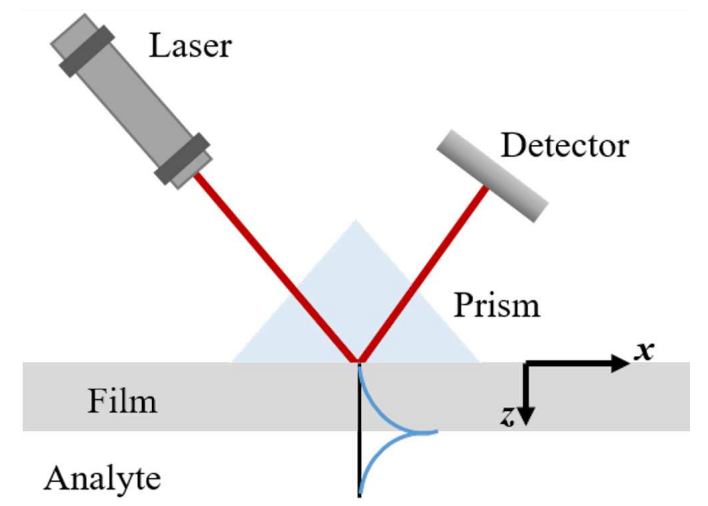

Fig. 1. The Kretschmann setup. Blue lines show profiles of resonantly excited fields. 


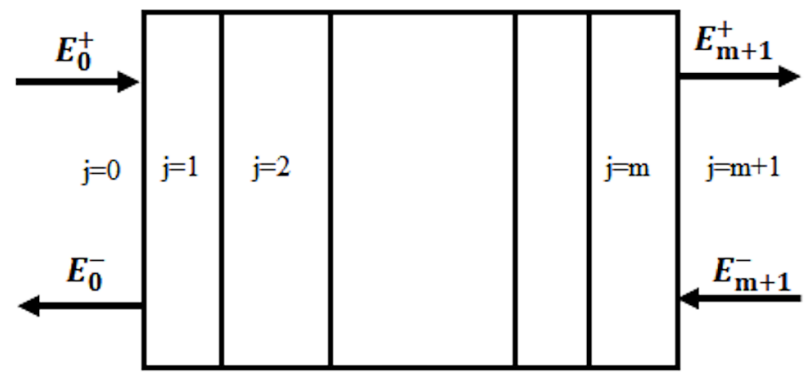

Fig. 2. Parameters of the numerical model: $E_{0}^{+}$and $E_{0}^{-}$are the incident amplitude of light's wave in the forward direction and the amplitude of light's wave propagating backwards from the system, respectively. $E_{m+1}^{+}$ and $E_{m+1}^{-}$are the forward transmitted amplitude of light's wave and the backwards propagating amplitude of light's wave, respectively. In most systems $E_{m+1}^{-}$is assumed to be 0 . If the layer index $j$ becomes $j=0$, then $j=m+1$ is assumed to be air.

The proposed numerical model uses the TMM and is based on complex amplitudes of Fresnel for the transmission and reflection at the boundary (interface). It would give an efficient prediction of the sensor response and will help to optimise the resolution of detection as well as the sensitivity.

Figure 2 shows the parameters used to model multilayered system. Each layer $j$ is described with an incidence angle $\theta_{j}$ and a thickness $h_{j}$, and can be characterised by the complex refractive index $n_{j}=n+\mathrm{i} k$. The incidence angle $\theta_{j}$ is related to the incidence angle in the system by relation

$$
\sin \left(\theta_{j}\right)=\frac{n_{0}}{n_{j}} \sin \left(\theta_{0}\right),
$$

where $n_{0}$ is the refractive index of the ambient medium. For each layer one deals with a $2 \times 2$ matrix, which is needed to find. One can use for it parameters $\beta_{i}$ and $p_{i}$, defined as:

$$
\begin{aligned}
& \beta_{j}=\frac{2 \pi}{\lambda} h_{j} n_{j} \cos \left(\theta_{j}\right), \\
& p_{j}=\frac{\cos \left(\theta_{j}\right)}{n_{j}} .
\end{aligned}
$$

The transfer matrix is derived directly from the Maxwell equations for macroscopic electromagnetism [14], where specific boundary conditions are used at each interface. To justify the use of the TMM in our model, some assumptions should be made. Firstly, each layer is considered infinitely wide as only its height will be taken into consideration. The interface only is assumed to be perfectly flat, while the transfer matrix method considers only homogeneous and isotropic films, we consider only plane waves which we assume incapable of handling diffusion between two adjacent layers. With these settings the transfer matrix of layer $j$ is written as

$$
\hat{M}_{j}=\left[\begin{array}{cc}
\cos \left(\beta_{j} h_{j}\right) & \frac{\mathrm{i}}{p_{j}} \sin \left(\beta_{j} h_{j}\right) \\
\mathrm{i} p_{j} \sin \left(\beta_{j} h_{j}\right) & \cos \left(\beta_{j} h_{j}\right)
\end{array}\right] .
$$

The full characteristic matrix of the system is constructed by multiplying the matrices $\hat{M}_{j}$ :

$$
\hat{M}=\prod_{j=2}^{N-1} \hat{M}_{j}
$$

The Fresnel coefficients for reflection $r$ and transmission $t$ for the whole system can then be extracted from

$$
\left[\begin{array}{c}
1+r \\
(-1+r) p_{0}
\end{array}\right]=\left[\begin{array}{c}
t \\
-t p_{m+1}
\end{array}\right] \text {. }
$$

In general, the characteristic matrix is described by its individual elements

$$
M=\left[\begin{array}{ll}
M_{11} & M_{12} \\
M_{21} & M_{22}
\end{array}\right]
$$

The conversion from the transfer matrix to the scattering matrix can be found from (6) and (7). The ATR is then the difference between the reflected energy $E_{0}^{-}$and the incident energy $E_{0}^{+}$. This can be easily see from the matrix elements $S$ and the total transmission given by [15]:

$$
\begin{aligned}
& S=\left[\begin{array}{ll}
S_{11} & S_{12} \\
S_{21} & S_{22}
\end{array}\right]=\left[\begin{array}{cc}
-\frac{M_{21}}{M_{22}} & \frac{1}{M_{22}} \\
M_{11}-\frac{M_{12} M_{21}}{M_{22}} & \frac{M_{12}}{M_{22}}
\end{array}\right], \\
& {\left[\begin{array}{l}
E_{0}^{+} \\
E_{0}^{-}
\end{array}\right]=\left[\begin{array}{ll}
S_{11} & S_{12} \\
S_{21} & S_{22}
\end{array}\right]=\left[\begin{array}{l}
E_{m+1}^{+} \\
E_{m+1}^{-}
\end{array}\right],}
\end{aligned}
$$

where $E_{0}^{+}, E_{0}^{-}, E_{m+1}^{+}$, and $E_{m+1}^{-}$relate to the energies shown in Fig. 2. This describes the internal scattering energies of the multi-layered system for which the reflection and transmission coefficients are

$$
\begin{aligned}
& R_{\mathrm{tot}}=|r|^{2}=\left|\frac{E_{0}^{-}}{E_{0}^{+}}\right|^{2}=\left|\frac{S_{21}}{S_{11}}\right|^{2}, \\
& T_{\mathrm{tot}}=|t|^{2}=\left|\frac{E_{m+1}^{-}}{E_{0}^{+}}\right|^{2}=\left|\frac{1}{S_{11}}\right|^{2} .
\end{aligned}
$$

Therefore, using this method for a set wavelength, layer thicknesses, and refractive indices, it is possible to sweep through the incident angle and reveal local minima in the spectra when the SPP conditions are met. This enables us to explore different structures to determine the response in each case. We begin from calculation of the change in refractive index of the sensed layer and the intensity of reflected light is plotted as function of the incidence angle. The angle corresponding to the minimum ATR changes when the refractive index changes indicating the plasmon resonance. The corresponding relative change in the refractive index is given by $[16]$ :

$$
\epsilon=\frac{\delta n}{n} \text {. }
$$

The scheme of the structure under consideration is shown in Fig. 3. A beam of light is transmitted through a prism of glass to the sensed layer (last medium). In between, a number of different layers of chromium, silver, gold, and graphene are used in order to realize a plasmon resonance and optimize the system sensitivity.

The complex refractive indices of different films used in the proposed configuration are summarized in Table I. 


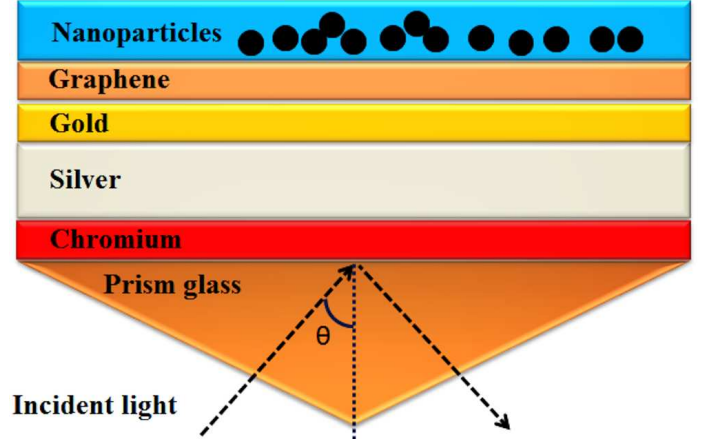

Fig. 3. The proposed configuration used to realize a surface plasmon resonance (SPR) sensor. The wavelength of the incident light is $750 \mathrm{~nm}$.

Complex refractive indexes of used films [17].

TABLE I

\begin{tabular}{c|c|c|c}
\hline \hline $\mathrm{Cr}$ & $\mathrm{Ag}$ & $\mathrm{Au}$ & $\mathrm{Gr}$ \\
\hline $3.84+4.37 \mathrm{i}$ & $0.146+4.908 \mathrm{i}$ & $0.164+4.35 \mathrm{i}$ & $3+1.361 \mathrm{i}$
\end{tabular}

\section{Plasmonic layers}

An active plasmonic layer is required to enable the plasmon resonance in SPR based sensor systems. The most common materials used as active plasmonic layers are gold and silver and this is due to their SPR appearing within the visible spectra [18].

Figure 4 shows the SPR response for configurations of glass/gold $(\mathrm{BK} 7 / \mathrm{Au})$ and for glass/silver $(\mathrm{BK} 7 / \mathrm{Ag})$ at a wavelength of $750 \mathrm{~nm}$. The configuration with silver shows a much narrower plasmon response (higher resolution) compared with gold, thereby, improving the sensitivity of the sensor [19]. Many studies have used gold for SPR biosensors [20, 21] due to its stable chemical and optical characteristics. Although, silver is not chemically stable but it can easily oxidize to form $\mathrm{AgO}_{2}$ which results in a degradation of the ATR signal [22].

Despite its stable chemical and optical characteristics, gold films have not proved to be efficient in developing highly sensitive SPR sensors, like silver films. To overcome the chemical instability of silver and develop a stable system with higher sensitivity, several attempts were made, including the insertion of a very thin layer of gold with the silver film [23]. However, this will modify the optimal thickness of silver film to obtain the best sensing sensitivity.

Figure 5 shows the ATR spectra obtained for the configuration $\mathrm{BK} 7 / \operatorname{Ag}(x \mathrm{~nm}) /$ water. We can see that increasing the Ag film thickness $x$ from $50 \mathrm{~nm}$ to $60 \mathrm{~nm}$ decreases the depth of the resonance peak. This means that the coupling efficiency of light into the surface plasmon mode on the silver film is reduced. It is due to the fact that the metal begins to act as a reflectance plane when its thickness increases to a point where light cannot couple to the surface charge oscillations which makes up

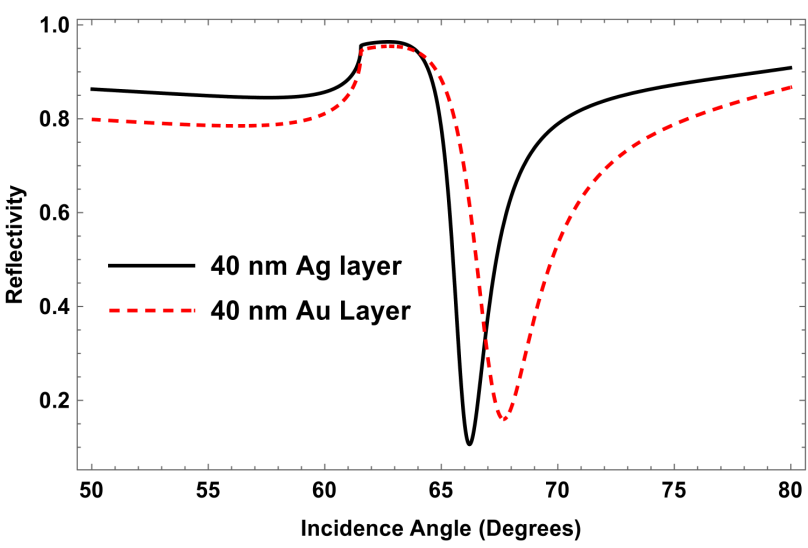

Fig. 4. Reflection intensity response for SPR system for $\mathrm{BK} 7 / \mathrm{Ag} /$ water (solid) and $\mathrm{BK} 7 / \mathrm{Au} /$ water (dashed) at a wavelength of $750 \mathrm{~nm}$. The thickness of the film is $40 \mathrm{~nm}$ for both cases.

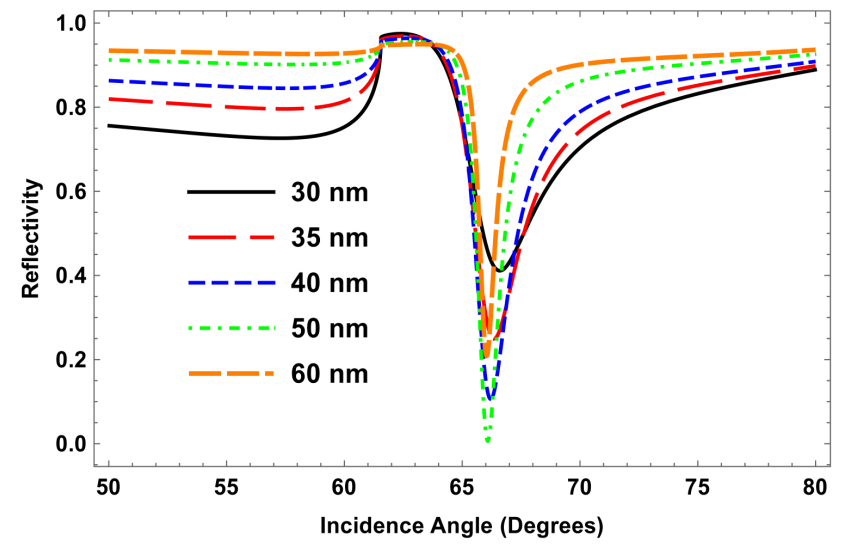

Fig. 5. The ATR as function of incidence angle for the configuration $\mathrm{BK} 7 / x /$ water at a wavelength of $750 \mathrm{~nm}$, where $x[\mathrm{~nm}]$ indicates different thickness values of the silver film.

the plasmon mode. However, when the silver film thickness is very thin $(30-35) \mathrm{nm}$, the coupling efficiency of light into the surface plasmon mode is more important. In this case, the sensitivity of the SPR system is reduced because the amplitude of the evanescent wave that penetrates into dielectric materials on the metal layer is higher than that of the surface plasmon that is generated from metal. Taking into account these effects, we find a compromise to build a satisfactory SPR system. Here, the optimal thickness to support an SPR system is around $40 \mathrm{~nm}$. For more convenience, Fig. 6 shows the variation of the ATR with incidence angle and the silver film thickness for the SPR configuration of Fig. 5.

It has been already reported [24-26] that group 1B metals like copper, silver, and gold have very poor adhesive properties in comparison to dielectric materials such as silicon dioxide $\left(\mathrm{SiO}_{2}\right)$, and therefore, they have a poor adhesion to glass substrates. To form a strong adhesion a transition metal such as chromium $(\mathrm{Cr})$ or 


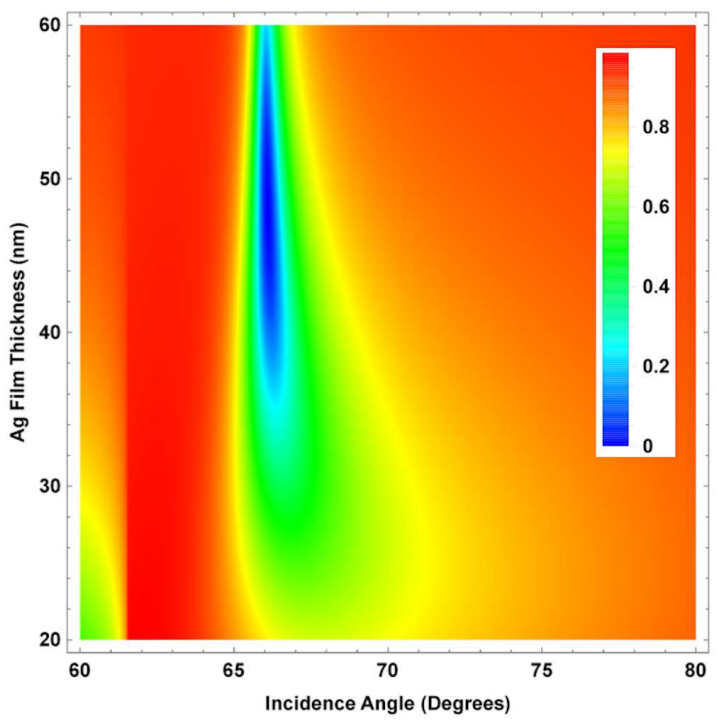

Fig. 6. The ATR as function of thickness $x$ of silver $\mathrm{Ag}$ layer obtained for SPR configuration $\mathrm{BK} 7 / \operatorname{Ag}(x \mathrm{~nm}) /$ water at a wavelength of $750 \mathrm{~nm}$.

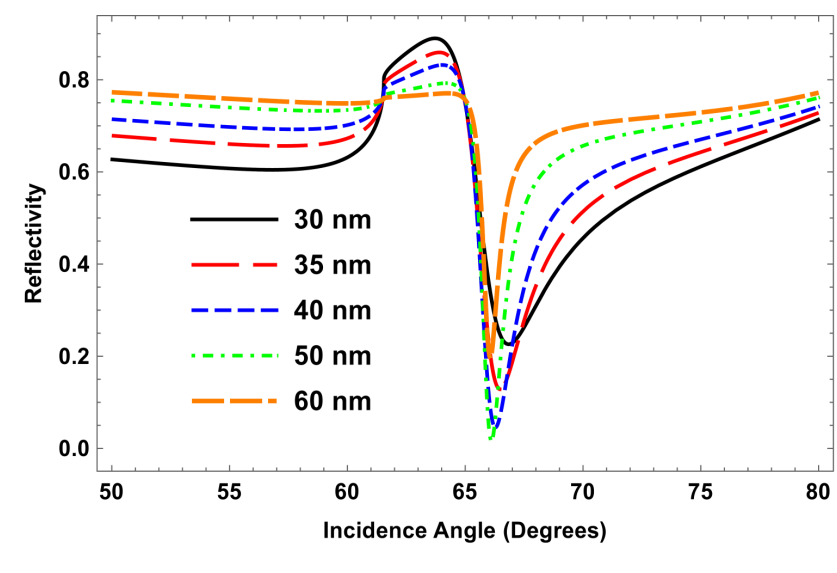

Fig. 7. The ATR spectra for a $\mathrm{BK} 7 / \mathrm{Cr}(2 \mathrm{~nm}) / \operatorname{Ag}(x \mathrm{~nm})$ system at a wavelength of $750 \mathrm{~nm}$. Values of $\mathrm{Ag}$ layer thickness $x$ are given in the legend.

titanium (Ti) can be used. A thin layer of chromium $(2-5) \mathrm{nm}$ has shown to form covalent oxide bonds at the surface with the hydroxyl groups causing much stronger adhesion $[24,26]$.

We introduce into our system a $2 \mathrm{~nm}$ chromium layer that the use changes the optimal thickness of the silver film. Figure 7 shows the ATR spectra for a configuration $\mathrm{BK} 7 / \mathrm{Cr} / \mathrm{Ag}(x \mathrm{~nm})$ for a range of different thicknesses values $x$ of $\mathrm{Ag}$ layer. Note that as the thickness of Ag layer increases, the SPR resonance peak becomes narrower (smaller FWHM). For a thickness greater than $50 \mathrm{~nm}$, the ATR signal becomes smaller.

Now we investigate parameters of the structure as the gold layer is introduced. Figure 8a and b shows the ATR spectra for the following configuration $\mathrm{BK} 7 / \mathrm{Cr}(2 \mathrm{~nm}) /$ $\mathrm{Ag}(40 \mathrm{~nm}) / \mathrm{Au}(x \mathrm{~nm}) /$ water. The thickness of $\mathrm{Cr}$ and
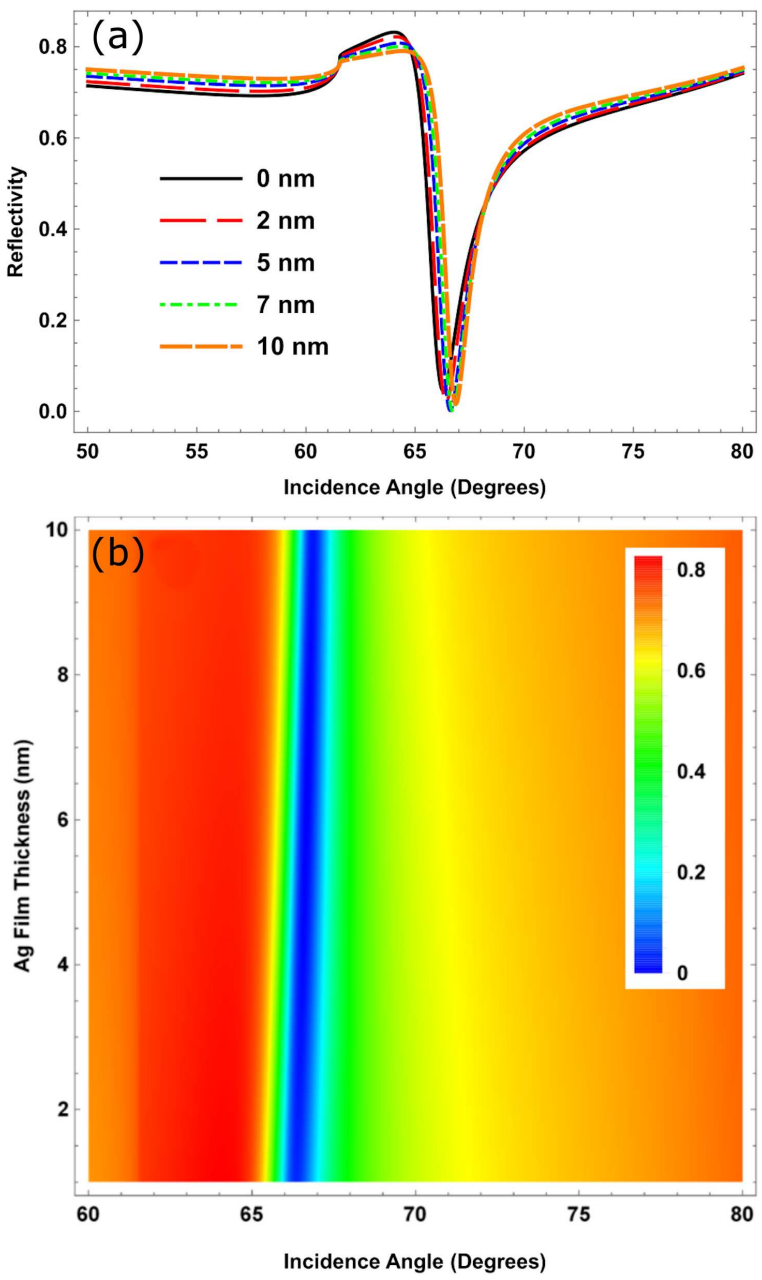

Fig. 8. The ATR spectra for the system $\mathrm{BK} 7 / \mathrm{Cr}(2 \mathrm{~nm}) / \mathrm{Ag}(40 \mathrm{~nm}) / \mathrm{Au}(x \mathrm{~nm})$ at a wavelength of $750 \mathrm{~nm}$. (a) Results are performed for selected $x$ values of gold layer thickness. (b) Results are a continuous function of $x$ values.

Ag layer is set to $2 \mathrm{~nm}$ and $40 \mathrm{~nm}$, respectively, while the thickness of gold layer, marked by $x$, is changed. As mentioned above in case of silver, the best thickness of gold is given by the deepest attenuation total reflection and narrowest FWHM of the resonance peak, which will result in high sensitivity and resolution of the system. The optimal thickness of gold film is around $5 \mathrm{~nm}$. It improves the sensor response when compared to just having the silver layer. This shows that the resonant angle and the ATR signal changes when the thicknesses of Ag layer changes.

It is known that molecules and particles at the sensing interface do not have a high affinity to gold. To avoid this problem and improve the affinity, an additional immobilization layer such as graphene is used at the interface. The addition of graphene significantly reduces the oxidisation of the metallic surfaces and provides a protective surface for the plasmonic layer [27]. One of the most 


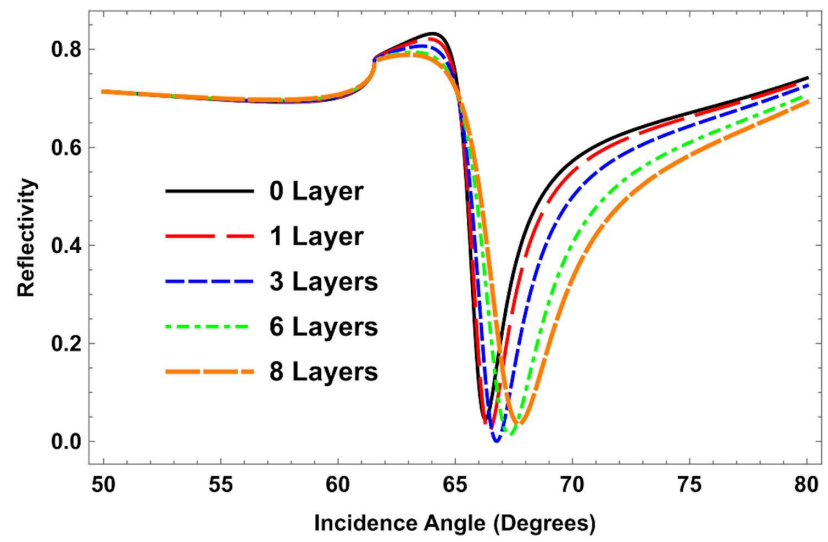

Fig. 9. The ATR signal obtained for the configuration $\mathrm{BK} 7 / \mathrm{Cr}(2 \mathrm{~nm}) / \mathrm{Ag}(40 \mathrm{~nm}) / \mathrm{Au}(5 \mathrm{~nm}) /$ graphene $(L$ layers), where $L$ is the number of graphene layers.

important parameters concerning the use of graphene in sensing applications is the complex refractive index. It determines the optical response and changes in the SPR conditions according to the number of graphene layers $L$. It can be calculated from the equation [28, 29]:

$$
\widetilde{n}=n+\mathrm{i} k=3+\frac{\mathrm{i}}{3} C_{g} \lambda,
$$

where $C_{g}=5.446 \mu \mathrm{m}^{-1}$ is given by the opacity measurements [30] and $\lambda$ is the wavelength in meter.

Another parameter that is needed for the simulations is the thickness of the graphene layers which is given by $d=L \times 0.34 \mathrm{~nm}[28]$. The percentage absorption per layer can also be estimated using the fine structure constant $a$, where the opacity is given as [30]:

$$
(1-T)=\pi a=\frac{\pi e^{2}}{\hbar c}=2.3 \%,
$$

where $e$ is the charge of the electron, $\hbar$ is the Planck constant $h$ divided by $2 \pi$, and $c$ is the speed of light.

The corresponding ATR signal is shown in Fig. 9 where configuration glass $/ \mathrm{Cr} / \mathrm{Ag} / \mathrm{Au}(x \mathrm{~nm}) /$ graphene/water is considered. The thickness of $\mathrm{Cr}, \mathrm{Ag}$, Au layer is $2 \mathrm{~nm}$, $40 \mathrm{~nm}, x \mathrm{~nm}$, respectively, and $L$ is the number of graphene layers. The introduction of graphene layer slightly increases the FWHM of the ATR and the resonance peak and also changes its depth. The SPR response is strongly dependent on the number of graphene layers and an optimum number of graphene layers can be used in order to get the highest sensitivity. The obtained results are very promising and highly encouraging. It is seen that for a given $\mathrm{Ag}$ thickness a deeper resonance requires an optimal number of graphene layers. In the case of $40 \mathrm{~nm}$ silver plasmonic layer the optimum number of graphene layers is $L=6$.

\section{Localized surface plasmon resonance sensor}

Localised surface plasmons (LSPs) are quantized plasma oscillations formed near the surfaces of metal nanoparticles. In contrast with propagating surface
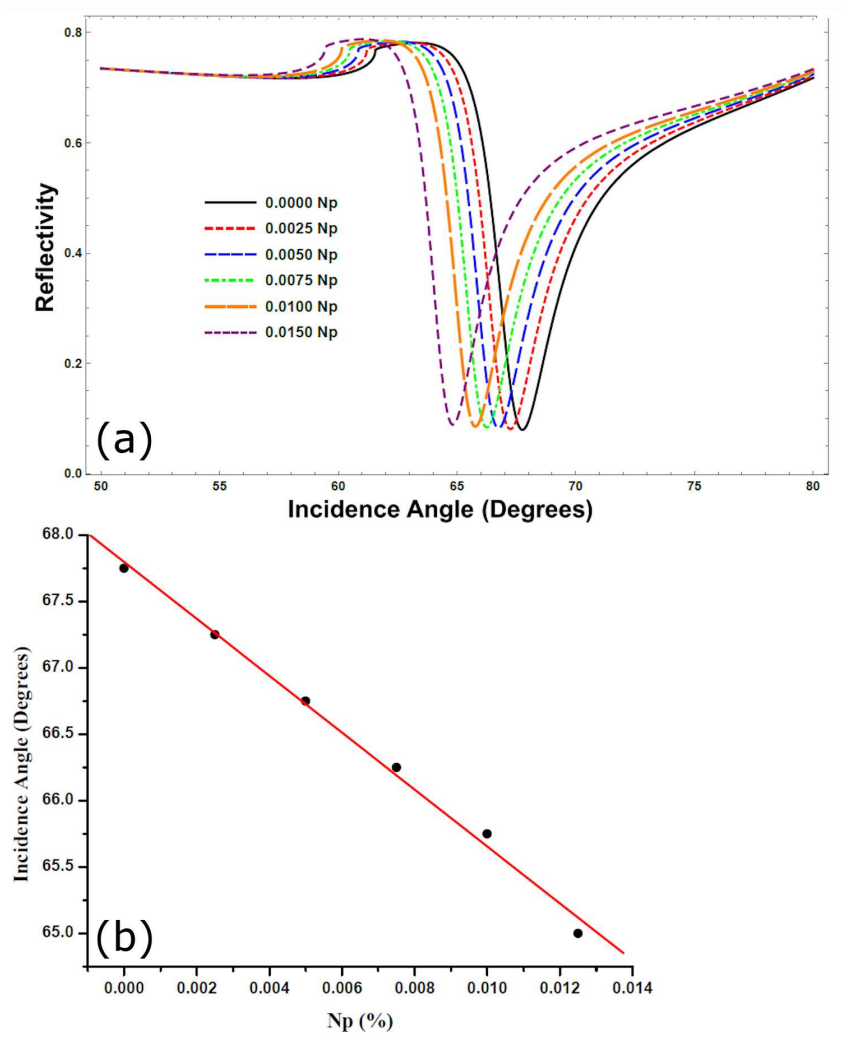

Fig. 10. (a) SPR response (ATR) as function of silver nanoparticles concentration for a $40 \mathrm{~nm}$ Ag silver plasmonic layer and 6 layers of graphene at a wavelength of $750 \mathrm{~nm}$. (b) Resonance angle (minimum of reflectivity) as a function of nanoparticles $(\mathrm{Np})$ concentration.

plasmons, which often require carefully-constructed optical arrangement for phase matching, LSPs can be excited easily by direct irradiation of light.

The curved surface of the particle effectively exerts a restoring force on the electron cloud, giving rise to a resonance. This implies that for frequencies close to the resonance frequency the optical response, i.e., the amplitude of the electron cloud, will be much larger than that away from the resonance. Additionally, since the electron cloud is charged and accelerating charges radiate, the fields both inside and outside the particle will be greatly amplified when the LSP is excited. This is because of the strong optical response and the large local field enhancement that the LSP resonance of metal nanoparticles has been the subject for a large application in surface-enhanced Raman scattering [31], fluorescence enhancement [32], refractive index (RI) measurement [33], biomolecular interaction detection [34], and so on. In particular, LSP can be used to sense individual particles without using plasmonic layers. However, if the particle size is much smaller than the wavelength, LSPR condition will not be satisfied. Yet, using the previous plasmonic system low concentrations of silver nanoparticles can be sensed since these particles change the refractive index of the sensed layer. Since we are interested in 
diluted systems, the permittivity of particles in aqueous solution can be evaluated within the effective medium theory [35]:

$$
\epsilon_{N}=\epsilon_{d}+4 \pi V \alpha
$$

where $\epsilon_{d}$ is the permittivity of the aqueous solution, $V$ is volume of the particle, and $\alpha$ is its dielectric polarizability which depends on the shape, size, and material of the used particle. Figure 10a shows the reflected intensity as functions of $\mathrm{Ag}$ particle concentration for plasmonic system $40 \mathrm{~nm} \mathrm{Ag} / 6$ layers of graphene. If the angle corresponding to the ATR minimum is plotted as a function of $\mathrm{Np}$ concentration the response is linear with a slope of $200^{\circ}$ / concentration (Fig. 10b).

\section{Discussion}

In this study, we proposed a robust and applicable model to detect metallic (conducting) nanoparticles. The particles of interest are silver nanoparticles, that are increasingly used in consumer products in spite of being highly toxic to aquatic life. The proposed model calculates the SPR response numerically using gold or silver as the plasmonic layer. It is shown that silver produces a sharper resonance response, however, to grow silver or gold onto glass requires a $(2-3) \mathrm{nm}$ adhesion layer which is in our case chromium $(\mathrm{Cr})$. This model investigates also the influence of the adhesion layer. To overcome the instability of silver, we use a $5 \mathrm{~nm}$ protective gold layer. Gold does not have a high affinity and the use of an optimum number of graphene layers is required due to its inherent electronic and optical as well as monoatomic thickness. The results are encouraging and shows that graphene layers have no effect on the sharpness of the SPR response. The thickness of the silver layer that produces the minimum reflectivity changes when we introduce graphene layers (without graphene, the thickness was $40 \mathrm{~nm}$, and with graphene it was reduced to $35 \mathrm{~nm})$. The numbers of layers of graphene were also varied and it was shown that increasing the number of layers has an important influence on the SPR response. Finally, we explored the detection of silver nanoparticles. It is well known that metallic nanoparticles with sizes much smaller than the wavelength are able to support localized plasmonic resonance. This property usually enhances SPR sensitivity, but here it was used to detect the particles themselves. The effective permittivity of water and small percentage of Ag particles were modeled and used to explore the response to increasing concentrations of Ag particles. The outcomes of this simulations revealed that there is a linear response to reflectivity with particle concentration.

\section{Conclusion}

A theoretical model for multiple layers of metals has been developed for SPR sensing. This analysis shows that silver offers better sensitivity than gold, but the latter is not reactive and therefore, is the material of choice in plasmonic sensing. We have also presented simulation results which demonstrate that functionalised graphene offers higher sensitivity and biomaterial affinity, due to its inherent electronic and optical as well as mono-atomic thickness. In addition, a new approach to improve sensitivity has also been proposed using a lock-in amplifier technique to measure intensity as well as the phase of the reflected optical signal. This, combined with the theoretical models detailed here, may potentially offer a route to single molecule detection.

\section{References}

[1] R.W. Wood, Philos. Mag. J. Sci. 4, 396 (2009).

[2] S. Stafford, R.S. Garcia, Y.K. Gunko, Appl. Sci. 8, 97 (2018).

[3] D.A. Stuart, A.J. Haes, C.R. Yonzon, E.M. Hicks, R.P. Van Duyne, IEE Proc. Nanobiotechnol. 152, 13 (2005).

[4] P.K. Jain, X. Huang, I.H. El Sayed, M.A. El-Sayed, Plasmonics 2, 107 (2007).

[5] N.E. Agbor, J.P. Cresswell, M.C. Petty, A.P. Monkman, Sens. Actuat. B Chem. 41, 137 (1997).

[6] K. Matsubara, S. Kawata, S. Minami, Appl. Opt. 27, 1160 (1988).

[7] B. Liedberg, I. Lundstrom, E. Stenberg, Sens. Actuat. B 11, 63 (1993).

[8] C. Nylander, B. Liedberg, T. Lind, Sens. Actuat. 3, 79 (1982).

[9] B. Liedberg, C. Nylander, I. Lundstrom, Sens. Actuat. 4, 299 (1983).

[10] L.M. Zhang, D. Uttamchandani, Electron. Lett. 23, 1469 (1988)

[11] J. Homola, I. Koudela, S.S. Yee, Sens. Actuat. B Chem. 54, 16 (1999).

[12] E. Kretschmann, H. Raether, Zeitschr. Naturforsch. A 23, 2135 (1968).

[13] V.M. Agranovich, D.L. Mills, Surface Polaritons: Electromagnetic Waves at Surfaces and Interfaces, North-Holland, Amsterdam 1982.

[14] M. Born, E. Wolf, Principles of Optics, CUP Archive, 1999.

[15] L.A.A. Pettersson, L.S. Roman, O. Inganäs, J. Appl. Phys. 86, 487 (1999).

[16] L.E. Kreno, J.T. Hupp, R.P. Van Duyne, Analyt. Chem. 82, 8042 (2010).

[17] E.D. Palik, Handbook of Optical Constants of Solids, Academic Press, 1998.

[18] S.A. Maier, Plasmonics: Fundamentals and Applications, Springer, 2007.

[19] S. Choi, K. Byun, J. Opt. Soc. Am. A 27, 2229 (2010).

[20] N. Maalouli, A.C. Gouget-Laemmel, B. Pinchemel, M. Bouazaoui, J.N. Chazalviel, F. Ozanam, Y. Yang, P. Burkhard, R. Boukherroub, S. Szunerits, Langmuir 27, 5498 (2011).

[21] S. Szunerits, X. Castel, R. Boukherroub, J. Phys. Chem. C 112, 15813 (2008).

[22] J. Yu, X. Yu, L. Zhang, H. Zeng, Sens. Actuat. B Chem. 173, 133 (2012). 
[23] E. Hutter, J.H. Fendler, D. Roy, J. Phys. Chem. B 105, 11159 (2001).

[24] P. Benjamin, C. Weaver, Proc. R. Soc. Lond. A 254, (1960).

[25] K.W. Vogt, P.A. Kohl, W.B. Carter, R.A. Bell, L.A. Bottomley, Surf. Sci. 301, 203 (1994).

[26] J.C. Hoogvliet, W.P. van Bennekom, Electrochim. Acta 47, 599 (2001).

[27] Y. Chen, H. Ming, Photon. Sens. 2, 37 (2012).

[28] L. Wu, H.S. Chu, W.S. Koh, E.P. Li, Opt. Express 18, 14395 (2010).

[29] S. Szunerits, N. Maalouli, E. Wijaya, J.P. Vilcot, R. Boukherroub, Anal. Bioanal. Chem. 405, 1435 (2013).
[30] R.R. Nair, P. Blake, A.N. Grigorenko, K.S. Novoselov T.J. Booth, T. Stauber, N.M.R. Peres, A.K. Geim, Science 320, 1308 (2008).

[31] C.Y. Chen, E. Burstein, Phys. Rev. Lett. 45, 1287 (1980).

[32] K. Sokolov, G. Chumanov, T.M. Cotton, Anal. Chem. 70, 3898 (1998)

[33] J. Zeng, D. Liang, Z.X. Cao, Proc. SPIE 17th Int. Conf. on Optical Fibre Sensors 5855, 667 (2005).

[34] R. Karlsson, A. Falt, J. Immunolog. Methods 200 121 (1997).

[35] C.A. Poland, R. Duffin, I. Kinloch et al., Nanotech 3 , 423 (2008). 